\title{
Modeling the gas-phase chemistry of the transitional disk around HD 141569A
}

\author{
B. Jonkheid ${ }^{1}$, I. Kamp ${ }^{2}$, J.-C. Augereau ${ }^{1,3}$, and E. F. van Dishoeck ${ }^{1}$ \\ 1 Sterrewacht Leiden, PO Box 9513, 2300 RA Leiden, The Netherlands \\ e-mail: jonkheid@strw. leidenuniv.nl \\ 2 Space Telescope Division of ESA, Space Telescope Science Institute, 3700 San Martin drive, Baltimore, MD 21218, USA \\ 3 Laboratoire d'Astrophysique, Observatoire de Grenoble, BP 53, 380431 Grenoble Cedex 9, France
}

Received 23 December 2005 / Accepted 14 March 2006

\begin{abstract}
Aims. The chemistry, distribution and mass of the gas in the transitional disk around the 5 Myr old B9.5 V star HD 141569A are constrained.

Methods. A quasi 2-dimensional (2D) chemistry code for photon dominated regions (PDR) is used to calculate the chemistry and gas temperatures in the disk. The calculations are performed for several gas distributions, PAH abundances and values of the total gas mass. The resulting CO $J=2-1$ and $J=3-2$ emission lines are computed with a 2D radiative transfer code and are compared to observations.

Results. The $\mathrm{CO}$ abundance is very sensitive to the total disk mass because the disk is in a regime where self-shielding just sets in. The observed $\mathrm{CO}$ emission lines are best fit by a power-law gas distribution of $80 M_{\oplus}$ starting at $80 \mathrm{AU}$ from the central star, indicating that there is some gas in the inner hole. Predictions are made for intensities of atomic fine-structure lines. [C I], which is the dominant form of carbon in large parts of the disk, is found to be a good alternative tracer of the gas mass.
\end{abstract}

Key words. astrochemistry - stars: individual: HD 141569A - stars: planetary systems: protoplanetary disks

\section{Introduction}

Circumstellar disks are the natural by-product of star formation, and once a protostar has formed they are crucial for the further accretion of matter onto the star (Shu et al. 1987). Over the course of its life a circumstellar disk loses its gas until a dusty debris disk remains in which the small dust grains are produced by collisions of planetesimals. The transformation from gas-rich accretion disks to dusty debris disks occurs relatively quickly (Simon \& Prato 1995), and as a result only a few objects in this transitional stage have been found. One example is the disk around HD 141569A, which is a pre-main sequence Herbig Ae/Be star with spectral type B9.5 V at a distance of 99 pc. It has a luminosity of $22 L_{\odot}$ (Merín et al. 2004). Its age is estimated at $\sim 5 \pm 3 \mathrm{Myr}$ (Weinberger et al. 2000; Merín et al. 2004) and it has two M-type companions HD 141569B and C (Rossiter 1943). The optically thin dust disk around HD 141569A has been observed in infrared emission (e.g. Sylvester et al. 1996) and in scattered light at near infrared (Augereau et al. 1999; Weinberger et al. 1999) and visible wavelengths (Mouillet et al. 2001; Clampin et al. 2003). Evidence for both large dust grains (Boccaletti et al. 2003) and polycyclic aromatic hydrocarbons (PAHs, see Sylvester et al. 1996; Weinberger et al. 2004) has been found. Gas in the disk has also been detected in the form of $\mathrm{CO}$ pure rotational lines (Zuckerman et al. 1995; Dent et al. 2005) and ro-vibrational lines (Brittain et al. 2003). Thus, this disk provides a unique laboratory to study the effects of dust evolution on the gas.
Observations of the dust in the HD 141569A disk show a complex morphology: there is a large inner hole in the dust distribution out to $150 \mathrm{AU}$ from the central star and two dust rings at $185 \mathrm{AU}$ and $325 \mathrm{AU}$ (Augereau et al. 1999; Weinberger et al. 1999; Mouillet et al. 2001; Boccaletti et al. 2003; Clampin et al. 2003). The outer dust ring may be explained by tidal interactions of the disk with the M-type companions (Augereau \& Papaloizou 2004), while both rings may also have formed due to hydrodynamic drag forces on the dust grains (Klahr \& Lin 2005 ) or interaction with a giant planet (Wyatt 2005). The origin of the large inner hole is still unclear but it could contain PAHs (Weinberger et al., in preparation).

The single dish CO observations by Zuckerman et al. (1995) and Dent et al. (2005) in principle provide constraints on the mass of gas in the disk. Since this object is in a transitional stage it should be losing its gas rapidly, and determining the gas mass and gas/dust ratio may give insight into the processes responsible for the gas loss. Additionally, the total amount of gas in the disk has implications on the dynamics of the dust grains, and an estimate of the gas mass and its distribution may help future dynamical simulations of the disk. In this paper a chemical model is presented which calculates molecular abundances at each position in the disk as well as line intensities to compare with submillimeter observations, building on the work by Kamp \& van Zadelhoff (2001), Kamp et al. (2003) and Jonkheid et al. (2004). By varying the gas distribution and the total gas mass of the disk, a best fit to the CO observations is presented. Predictions for other gas tracers are made. 

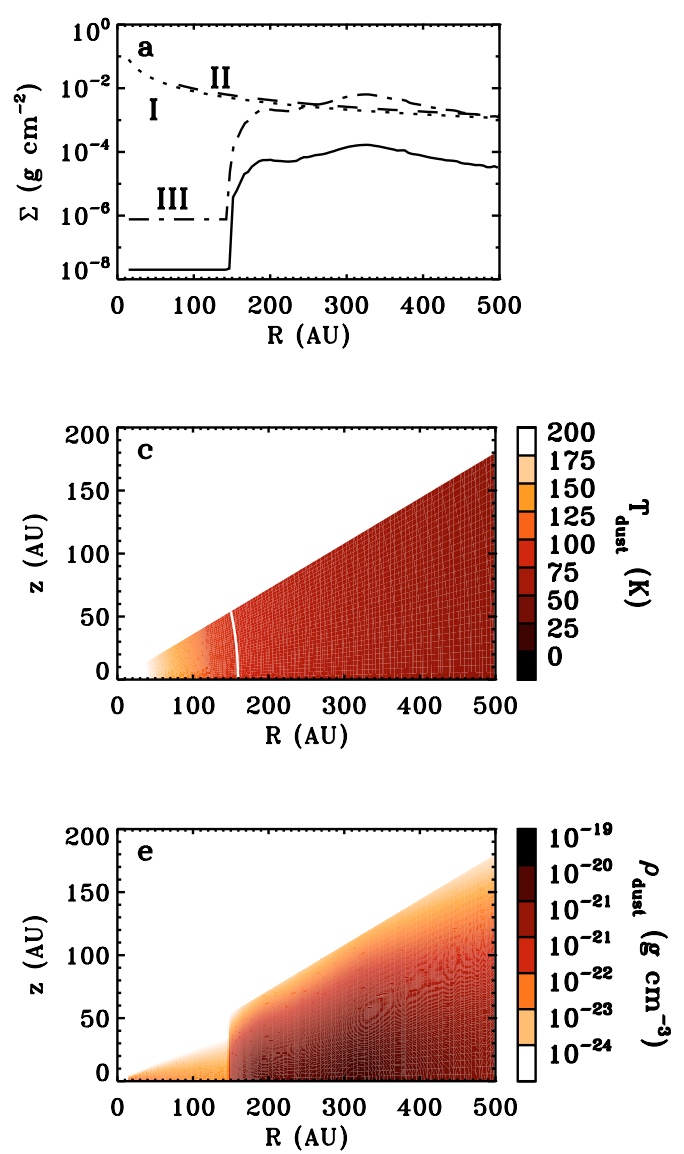
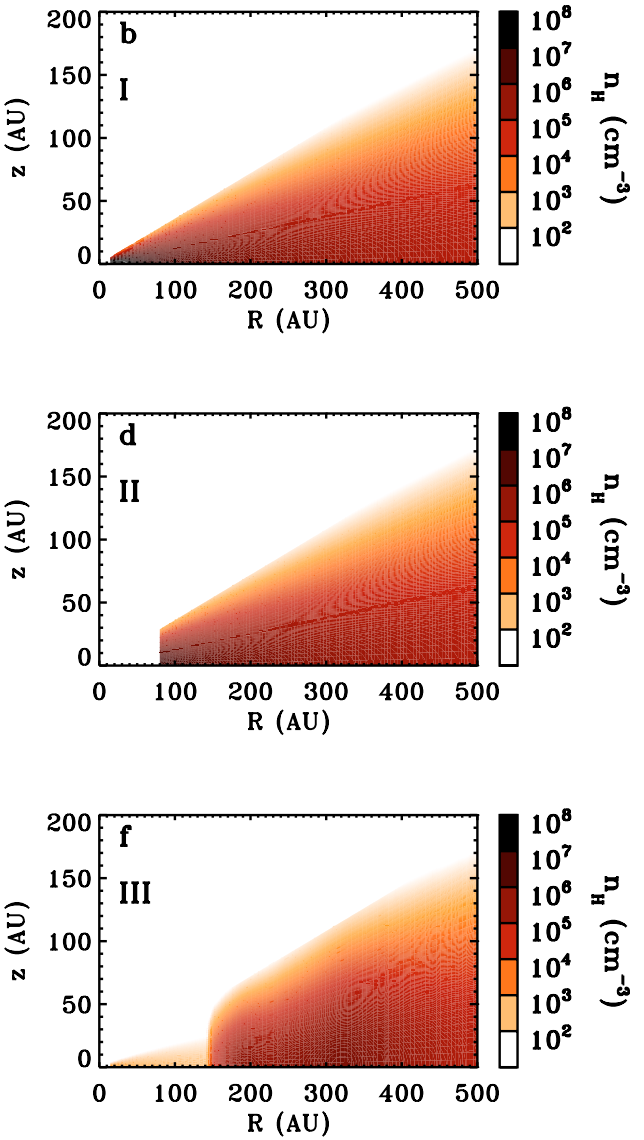

Fig. 1. Input parameters of the disk model. Panel a) gives the surface densities for dust (solid line) and for three different distributions of gas (dashed lines). Panels b), d) and f) give the corresponding gas number densities. Panel c) gives the mean dust temperature (averaged over the grain size distribution), where the white line denotes the $100 \mathrm{~K}$ isotherm. Panel e) gives the mass density of dust grains.

Table 1. Adopted gas-phase elemental abundances with respect to hydrogen.

\begin{tabular}{ll}
\hline \hline Element & Abundance \\
\hline $\mathrm{C}$ & $1.3 \times 10^{-4}$ \\
$\mathrm{O}$ & $2.9 \times 10^{-4}$ \\
$\mathrm{Mg}$ & $4.2 \times 10^{-6}$ \\
$\mathrm{~S}$ & $1.9 \times 10^{-6}$ \\
$\mathrm{Si}$ & $8.0 \times 10^{-6}$ \\
$\mathrm{Fe}$ & $4.3 \times 10^{-6}$ \\
$\mathrm{PAH}$ & $1-2 \times 10^{-10 a}$ \\
\hline
\end{tabular}

${ }^{a}$ The PAH abundance varies between models with different gas masses, since the total mass in PAHs is kept constant.

\section{Model}

\subsection{Input}

The basis for the calculations presented here is formed by the 1+1-dimensional model described in Jonkheid et al. (2004). In this model the disk is divided into a series of vertical 1-dimensional structures, each of which was treated as a photon dominated region (PDR) illuminated from above. The PDR code calculates the chemistry using the detailed radiative transfer of $\mathrm{H}_{2}$ and $\mathrm{CO}$ dissociating lines described by Black \& van Dishoeck (1987) and van Dishoeck \& Black (1988). The elemental abundances used in the calculations are displayed in Table 1; it is assumed that the gas in the disk is of interstellar origin instead of second generation gas such as may result from the evaporation of solid bodies. The thermal balance is solved taking into account thermal coupling between gas and dust, heating through photoionization of polycyclic hydrocarbons (PAHs), photoelectric effect on large grains, and the formation and dissociation of $\mathrm{H}_{2}$ and cooling through $\mathrm{CO}$ rotational lines and the fine-structure lines of $\mathrm{C}, \mathrm{C}^{+}$and $\mathrm{O}$. The code was tested extensively against other codes in a benchmark project (Roellig et al., in preparation).

Since the HD 141569A disk differs significantly in terms of mass and structure from the T-Tauri disks examined in Jonkheid et al. (2004), a different input is used (see Fig. 1). The dust distribution is taken from Mouillet et al. (2001) by deprojecting the disk assuming a scattering phase function with $g=0.2$ in the $V$-band (see Fig. 2). The dust content in the inner hole is uncertain: the upper limit to the surface density is $2 \times 10^{-6} \mathrm{~g} \mathrm{~cm}^{-2}$. In the calculations a value of $2 \times 10^{-8} \mathrm{~g} \mathrm{~cm}^{-2}$ is used at $R<$ $150 \mathrm{AU}$ to be on the conservative side. An azimuthal average of the distribution was then taken to obtain the surface density in Fig. 1a.

Several trial profiles were used for the radial gas distribution: a power law similar to the minimal mass solar nebula where $\Sigma_{\text {gas }} \propto R^{-1.2}$, with an inner radius of either 15 AU (called distribution I, Fig. 1b) or 80 AU (distribution II, Fig. 1d), and a distribution similar to the dust distribution (with an effective inner radius of $150 \mathrm{AU}$, called distribution III, see Fig. 1f). For each assumption of the radial distribution the total gas mass was varied by scaling the entire distribution by a single factor. The densities of gas and dust were derived from their surface densities 


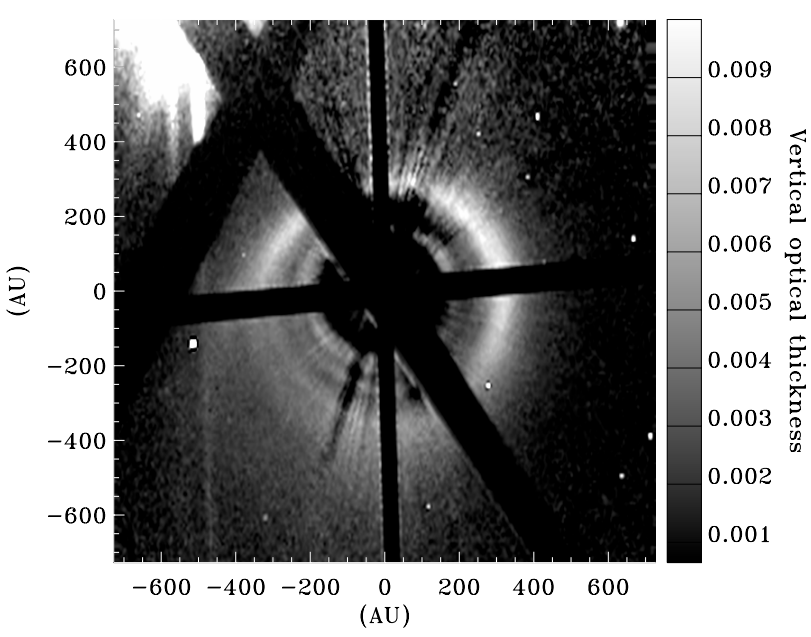

Fig. 2. Deprojected HST image of the HD 141569 disk in scattered light by Mouillet et al. (2001).

assuming a vertical distribution of $n \propto \mathrm{e}^{-z^{2} / 2 h^{2}}$, where the scale height $h=0.085 R$ and $R$ and $z$ are cylindrical coordinates. The densities shown in Fig. 1 for the different radial distributions are for total gas masses of $80 M_{\oplus}$. The dust temperature is determined assuming the grains are in thermal balance and the disk is optically thin in continuum radiation at infrared through ultraviolet (UV) wavelengths.

The interstellar spectrum used for the UV radiation by Jonkheid et al. (2004) is inappropriate for HD 141569 in the chemically important wavelength range of $912 \AA<\lambda<1100 \AA$, so the spectrum of an A0 star (taken from Hauschildt et al. 1999, with $T_{\text {eff }}=10000 \mathrm{~K}$ and $\log g=4$, see Fig. 3) was used here to calculate the photoionization and photodissociation rates. For the photoelectric heating rate the differences in the spectral shape of the radiation field were implemented using the correction factor by Spaans et al. (1994) (Eq. (13) in that paper). The radiation field has a strength of $I_{\mathrm{UV}}=10^{7} \times$ the strength of the interstellar radiation field by Draine (1978) at 15 AU from the central star; at $80 \mathrm{AU}$ this factor is $3 \times 10^{5}$.

Jonkheid et al. (2004) considered only more massive, optically thick disks, so a number of changes had to be made in the code to simulate this transitional object. First, the vertical PDR structures used in the original code are inappropriate here because scattering will be far less important in an optically thin disk. Therefore the PDRs are now taken radially from the star, with the strength of the radiation field scaling with $r^{-2}$ (where $r$ is the distance to the star, not to be confused with $R$, the radial component of the cylindrical coordinate system used in the plots) in addition to absorption effects.

Second, the absorptions responsible for dissociation of $\mathrm{H}_{2}$ and $\mathrm{CO}$ and the ionization of $\mathrm{C}$ may become optically thick if the column densities of these species toward the central star become sufficiently high. The interstellar radiation field then becomes the most important source of dissociation of these molecules. This is implemented in the model by taking the photodissociation rates for these molecules to be:

$R_{\mathrm{ph}, \text { tot }}=R_{\mathrm{ph}, \mathrm{star}}+R_{\mathrm{ph}, \mathrm{ISM}}$

where $R_{\mathrm{ph}, \text { tot }}$ is the total photorate, $R_{\mathrm{ph} \text {, star }}$ is the photorate due to stellar light and $R_{\mathrm{ph} \text {, ISM }}$ is the photorate due to the interstellar radiation field. The former rates are calculated using the detailed treatments of Black \& van Dishoeck (1987) for $\mathrm{H}_{2}$, van Dishoeck \& Black (1988) for CO and van Dishoeck (1988)

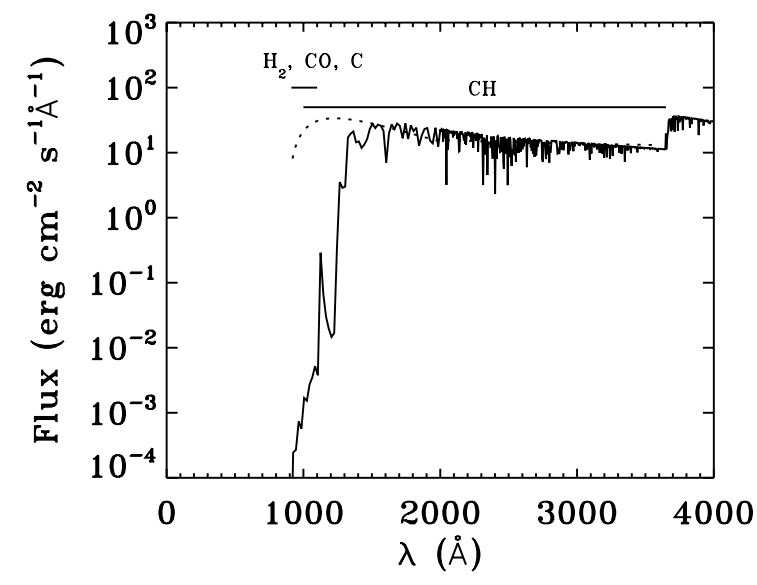

Fig. 3. The flux at the inner edge of the gas disk in distribution I and II (15 AU from the central star), for the radiation field of a B9.5 star (solid line) and of the interstellar radiation field (dotted line), scaled to fit the integrated flux between 912 and $3600 \AA$, corresponding to $I_{\mathrm{UV}}=$ $10^{7}$ times the interstellar radiation field. The spectral regime where $\mathrm{H}_{2}$ and $\mathrm{CO}$ are dissociated and $\mathrm{C}$ ionized, and the regime where $\mathrm{CH}$ is dissociated are indicated with bars at the top.

for $\mathrm{C}$. The latter rates are calculated assuming they have a value of $50 \%$ of the unshielded interstellar photodissociation rate at the disk's surface, with shielding calculated using the vertical column densities of $\mathrm{H}_{2}, \mathrm{CO}$ and $\mathrm{C}$ toward the nearest surface as inputs for the shielding functions by Draine \& Bertoldi (1996) for $\mathrm{H}_{2}$, van Dishoeck \& Black (1988) for CO and Werner (1970) for $\mathrm{C}$. For all other processes the interstellar radiation field was ignored, because the stellar radiation field has a strength of $I_{\mathrm{UV}}>1000 \times$ the interstellar UV field of Draine (1978) (disregarding absorption) even at $500 \mathrm{AU}$ and dominates the continuum flux.

\subsection{Treatment of dust}

Based on the scattering properties investigated by Boccaletti et al. (2003) and Augereau \& Papaloizou (2004) and the SED fitting by Li \& Lunine (2003) the dust particles in the HD 141569A disk are found to be much larger than their counterparts in the interstellar medium: HD 141569 dust has grain sizes $1 \mu \mathrm{m}<a<$ $1 \mathrm{~cm}$ (with $a$ the equivalent spherical radius), and a size distribution $n(a) \propto a^{-3.3}$ (Li \& Lunine 2003), while the ISM grains have sizes $5 \mathrm{~nm}<a<0.25 \mu \mathrm{m}$ and a distribution $n(a) \propto a^{-3.5}$ (Mathis et al. 1977). Since the Leiden PDR code assumes dust grains to have an interstellar size distribution, it has to be modified to incorporate the larger sized grains. Dust grains enter the model in four ways: as absorbers for UV radiation, as sites for $\mathrm{H}_{2}$ formation, and in the thermal balance as a source for the photoelectric heating and gas-dust collisions. These processes are, to lowest order, proportional to either the surface area or the geometrical cross-section of the grains, so they can be approximated by scaling them with

\section{$\left\langle a^{2}\right\rangle_{\mathrm{HD} 141569} /\left\langle a^{2}\right\rangle_{\mathrm{ISM}}$}

where $\left\langle a^{m}\right\rangle=\int_{a_{\min }}^{a_{\max }} a^{m} n(a) \mathrm{d} a / \int_{a_{\min }}^{a_{\max }} n(a) \mathrm{d} a$, and assuming the number of grains to remain constant in a given volume. If the mass in grains is kept constant rather than the number, all rates have to be mutiplied with a further factor of

$$
\frac{m_{\text {grain,ISM }}}{m_{\text {grain,HD } 141569}}=\frac{\left\langle a^{3}\right\rangle_{\text {ISM }}}{(1-P)\left\langle a^{3}\right\rangle_{\text {HD } 141569}}
$$



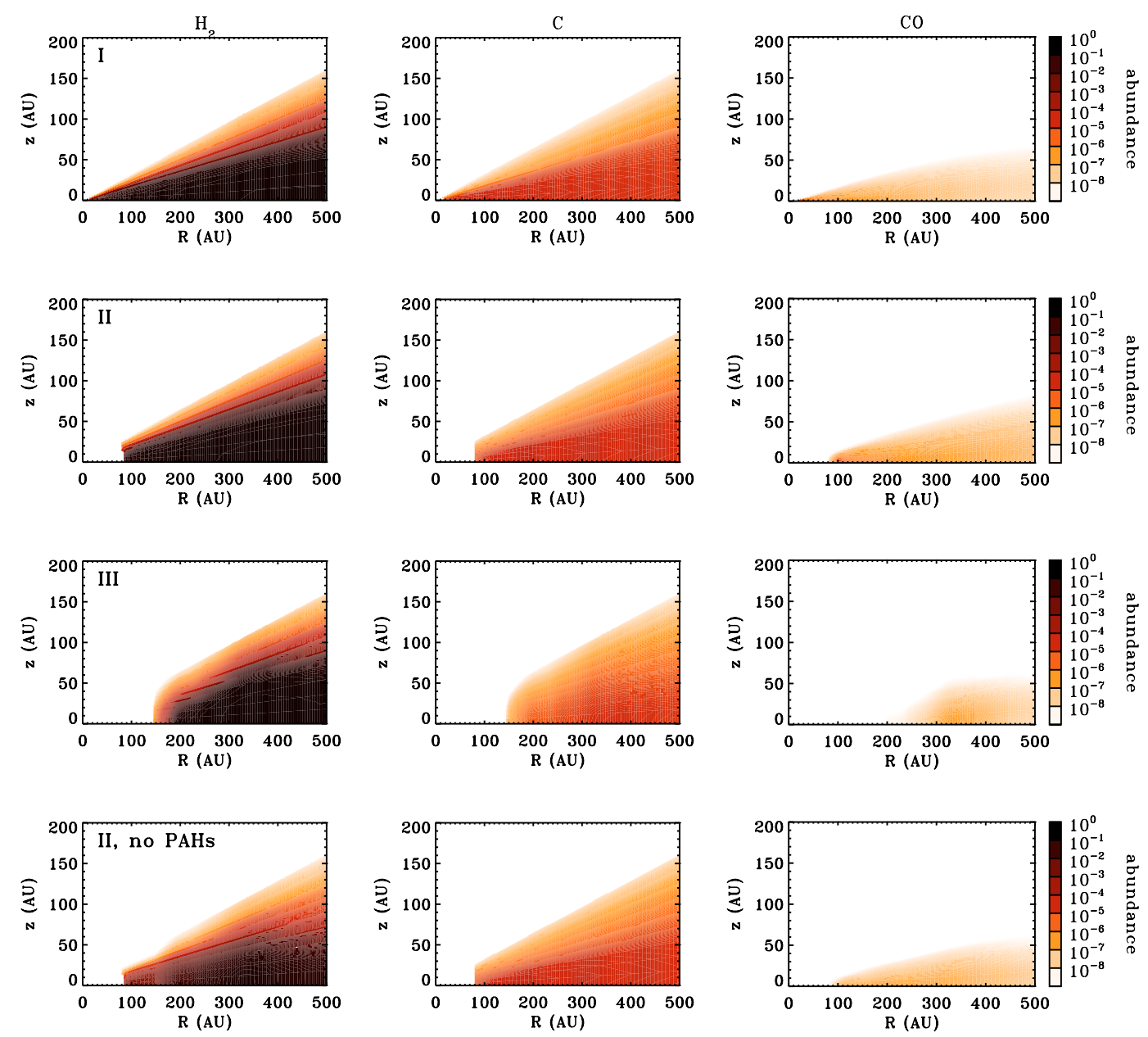

Fig. 4. Abundances (per $\mathrm{H}$ atom) for $\mathrm{H}_{2}$ (left column) $\mathrm{C}$ (middle column) and $\mathrm{CO}$ (right column) for models I (first row), II (second row), III (third row) and II without PAHs (fourth row). In all cases the gas mass is $80 M_{\oplus}$.

where $P$ is the porosity of the grains in the HD 141569A disk. Following Li \& Lunine (2003), $P=0.73$ for ice-covered grains. Since most of the dust has a temperature lower than $100 \mathrm{~K}$, this value is used everywhere in the disk. Thus, to lowest order all processes should be scaled by a factor $1 / 1500$ using the grain parameters derived for HD 141569 for a gas/dust mass ratio of 100.

This treatment of the dust properties leaves some inaccuracies of order unity, the most important ones being in the extinction of stellar radiation and the photoelectric heating rate. The mean extinction cross sections and scattering properties were calculated independently using Mie theory with the dielectric function of Li \& Lunine (2003) for porous icy aggregates (Eq. (9) in that paper); the results were used to correct the extinction. The photoelectric heating rate was calculated using a photoelectric yield of $Y=0.05$ electrons/photon to simulate larger grains (Watson 1972). For a single grain size of $3 \mu \mathrm{m}$ the resulting heating rates are similar to those used by Kamp \& van Zadelhoff (2001); for the HD 141569 grains the appropriate size distribution was used.

Even though the mean dust temperature shown in Fig. 1 is high enough to prevent freeze-out of $\mathrm{CO}$ on the grains, there remains the possibility that the large grains, which have a temperature lower than the mean dust temperature, act as sites for $\mathrm{CO}$ freezing. Calculations show that the temperature of $>100 \mu \mathrm{m}$ sized grains falls below $30 \mathrm{~K}$ only in the outer regions of the disk, and that even millimeter sized grains have temperatures $>25 \mathrm{~K}$ throughout the disk. It is therefore unlikely that freeze-out of $\mathrm{CO}$ will occur at any significant rate, so it is ignored in the calculations.

From infrared observations by Sylvester et al. (1996) it is known that polycyclic hydrocarbons (PAHs) are present in the disk. In our model PAHs are assumed to be well mixed with the gas, i.e. the abundance of PAH molecules with respect to hydrogen is independent of location in the disk, even in the inner hole. The total amount of PAHs is kept constant at $m_{\mathrm{PAH}}=7.9 \times$ $10^{-6} M_{\oplus}$ (Li \& Lunine 2003), regardless of the total gas mass. This means that the PAH abundance varies between models with different mass; their abundance is $x_{\mathrm{PAH}}=1.5 \times 10^{-10}$ per $\mathrm{H}$ atom when $M_{\text {gas }}=80 M_{\oplus}$. PAHs affect the disk's chemistry in three ways (see Jonkheid et al. 2004): as sources of photoelectric heating, as absorbers of UV radiation and by direct chemical reactions (particularly in the formation of $\mathrm{H}_{2}$ and the charge balance). These processes are not only important in the inner disk where practically no large grains are present, but also in the outer disk due to the decreased efficiency of the large grains.

\section{Results}

\subsection{Chemistry}

The results for the chemistry for models with different radial gas distributions are shown in Figs. 4 and 5. It can be seen that models I and II show similar behaviour, with molecular abundances that are high at the inner edge of the disk. Model III on the other 

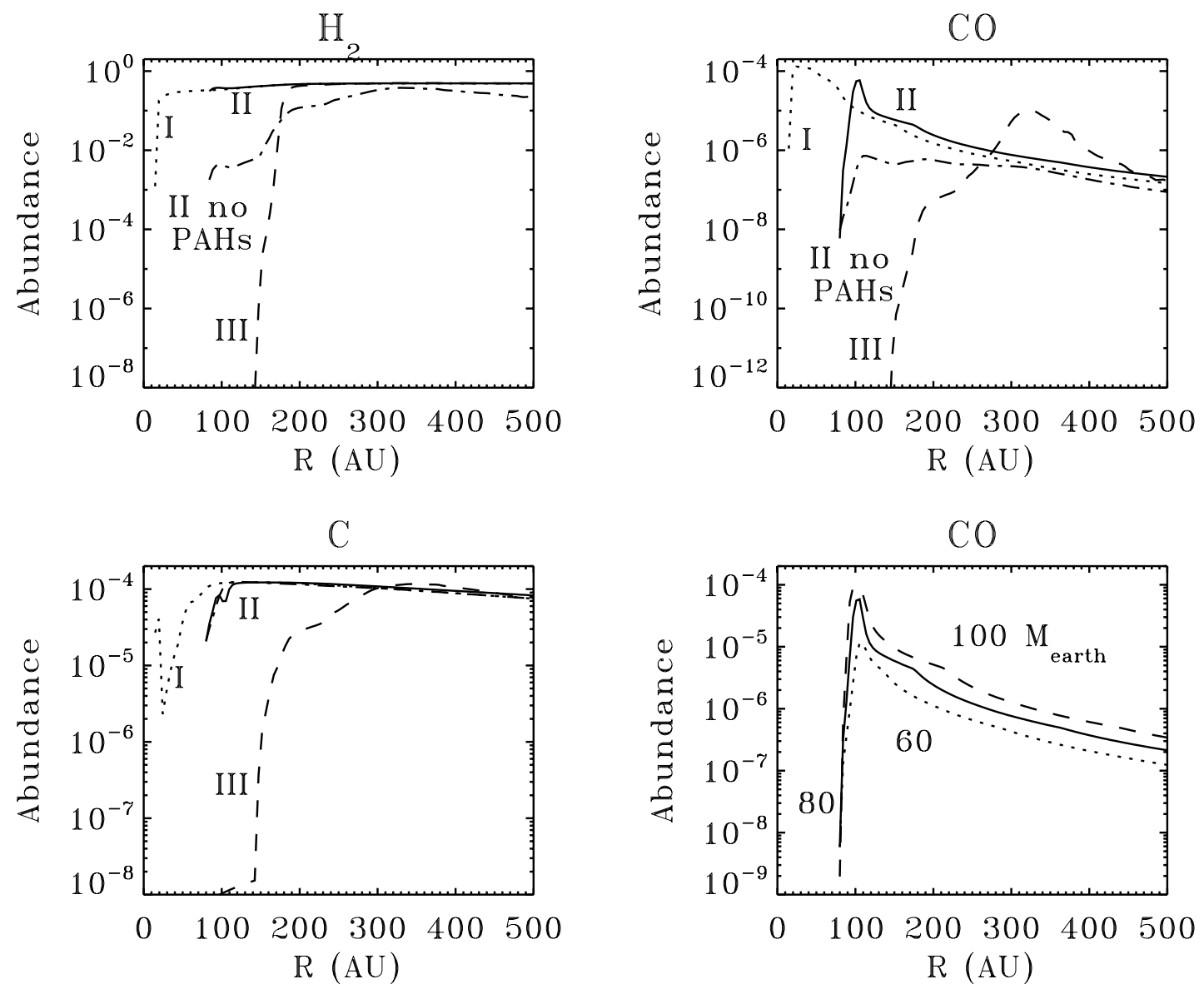

Fig. 5. Midplane abundances (per $\mathrm{H}$ atom) for $\mathrm{H}_{2}$ (upper left), $\mathrm{CO}$ (upper right) and $\mathrm{C}$ (lower left) for $80 M_{\oplus}$ gas distributions I (dotted line), II (solid line) and III (dashed line), and for distribution II when PAHs are removed from the chemistry (dash-dotted line). The lower right panel gives the midplane abundance of CO for gas distribution II with total gas masses of 60 (dotted line), 80 (solid line) and $100 M_{\oplus}$ (dashed line).

hand produces $\mathrm{H}_{2}$ and $\mathrm{CO}$ only very far in the outer regions of the disk. In all models the distribution of atomic carbon resembles the $\mathrm{H}_{2}$ distribution.

It can be seen in Fig. 5 that in most models the carbon in the midplane is mostly atomic rather than ionized or molecular (in the form of $\mathrm{CO}$ ). The reason for this is that even though the radiation responsible for the photoionization of $\mathrm{C}$ and the photodissociation of $\mathrm{CO}$ is largely shielded by the upper layers, there are still many photons with $\lambda>1200 \AA$ left over (see Fig. 3). These photons can proceed to dissociate precursors to $\mathrm{CO}$ (most notably $\mathrm{CH}$ ), thereby decreasing the $\mathrm{CO}$ formation rate and thus driving the carbon chemistry to a neutral atomic phase.

In Fig. 5 the $\mathrm{CO}$ abundances in the midplane are also shown for model II with total gas masses of 60,80 and $100 M_{\oplus}$. It can be seen that the $\mathrm{CO}$ abundance depends strongly on the gas mass, since it changes by an order of magnitude with only a factor of 2 change in mass. This strong dependence on mass is due to selfshielding; in this regime shielding of $\mathrm{CO}$ just sets in, and therefore every small increase in $\mathrm{CO}$ abundance in the upper layers enhances the production of $\mathrm{CO}$ in lower layers, producing a positive feedback loop.

Throughout the disk $\mathrm{H}_{2}$ is primarily formed on PAHs, and the large dust grains are very inefficient absorbers of UV radiation. The chemical structure therefore shows no correlation with the dust distribution. If PAHs are removed from the chemistry $\mathrm{H}_{2}$ is primarily formed on large grains, and its abundance resembles the dust distribution. Because of the decreased efficiency of $\mathrm{H}_{2}$ formation on the large grains the molecular abundances are lower than when PAHs are present. This is especially true for $\mathrm{CO}$, which now cannot build up sufficient column densities to become self-shielding.

It can be seen from Fig. 6 that the contribution of the interstellar UV field to the photodissociation of $\mathrm{H}_{2}$ and $\mathrm{CO}$ and the photoionization of $\mathrm{C}$ can be more than a factor of 100 higher than the stellar contribution in the outer disk, and therefore cannot be ignored in chemical calculations. This is especially true for $\mathrm{C}$ ionization, since the high abundance of atomic carbon in the inner disk shields the outer disk from the stellar radiation. The abundance of $\mathrm{H}_{2}$ is high enough for this molecule to become completely self-shielding in the vertical direction as well as the radial direction. In the case of $\mathrm{CO}$ and $\mathrm{C}$, the vertical selfshielding only just sets in. Thus the relative contribution of the interstellar UV field to $\mathrm{H}_{2}$ dissociation is not as large as it is for $\mathrm{CO}$ dissociation and $\mathrm{C}$ ionization.

The overall chemical timescales, which are controlled by the photorates, are short, ranging from from $<100 \mathrm{yr}$ in the inner disk and surface layers to a few $\times 10^{3} \mathrm{yr}$ in the midplane of the outer disk. Thus chemical equilibrium is attained on dynamical timescales.

\subsection{Temperature}

The results for the gas temperature are shown in Fig. 7. For models I and II the surface layers of the disk are cool $(\sim 20 \mathrm{~K})$, because the photoelectric heating by PAHs and large grains is 

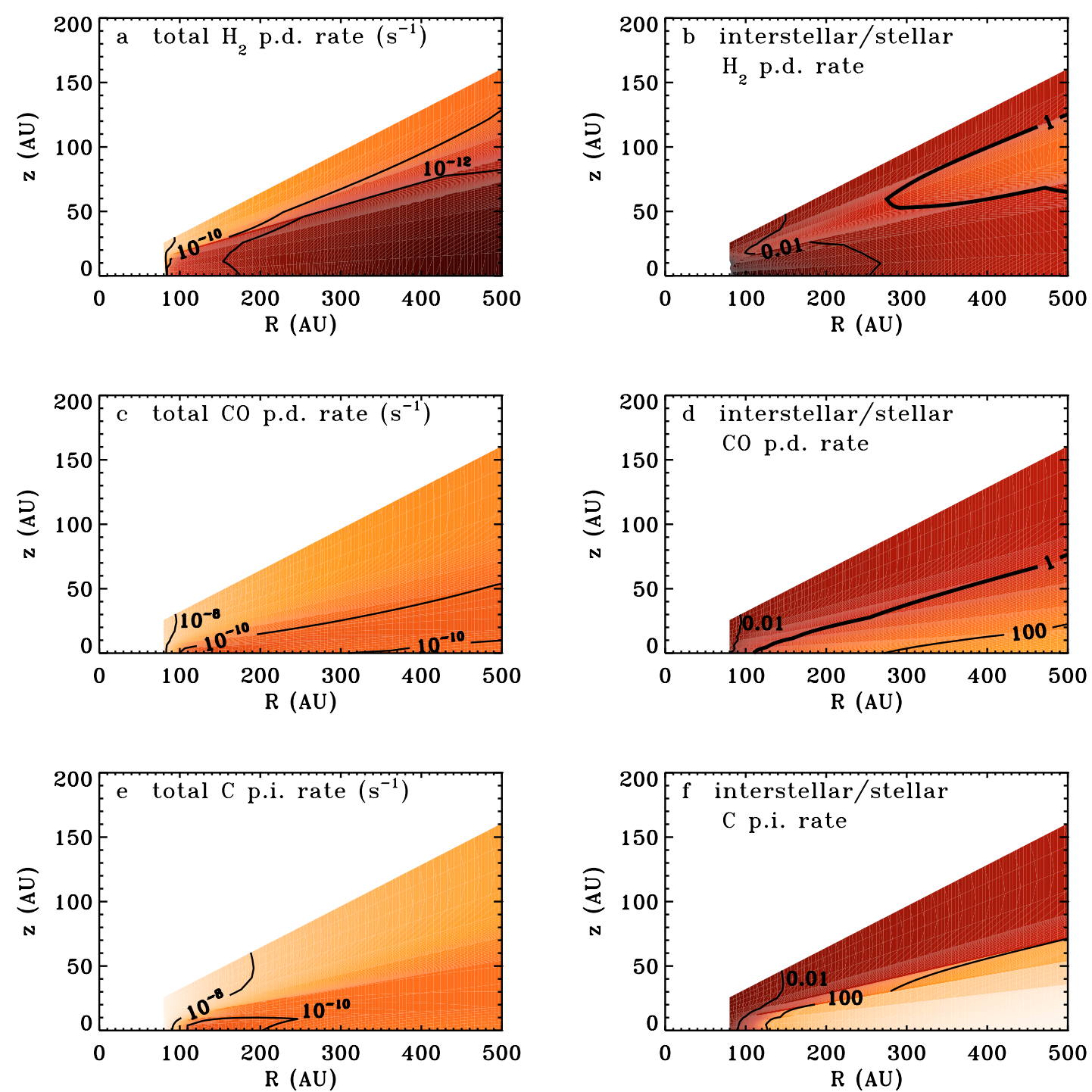

Fig. 6. The total photodissociation rates of $\mathrm{H}_{2}$ (panel a)) and $\mathrm{CO}$ (panel c)) and the total photoionization rate of $\mathrm{C}$ (panel e)) in model II, and the ratio of the contibutions of the interstellar and stellar radiation fields (panels $\mathbf{b}$ ), $\mathbf{d}$ ) and $\mathbf{f}$ )). Results for models with different gas masses follow similar qualitative trends.
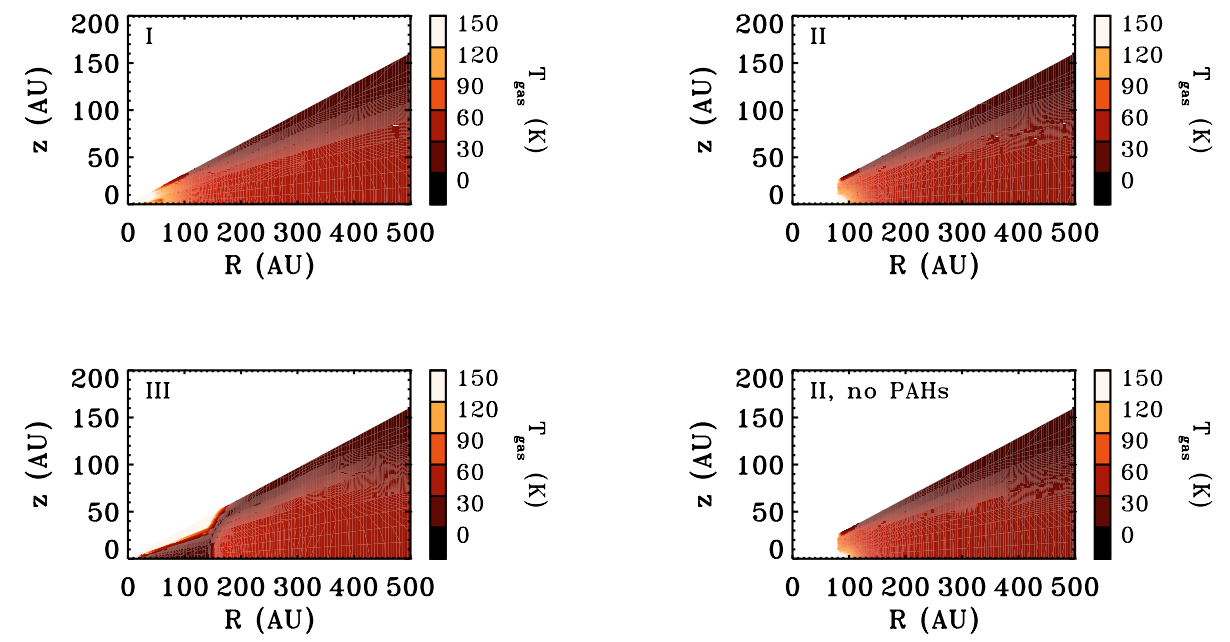

Fig. 7. The gas temperatures found for models I (upper left), II (upper right), III (lower left) and II without PAHs (lower right). 

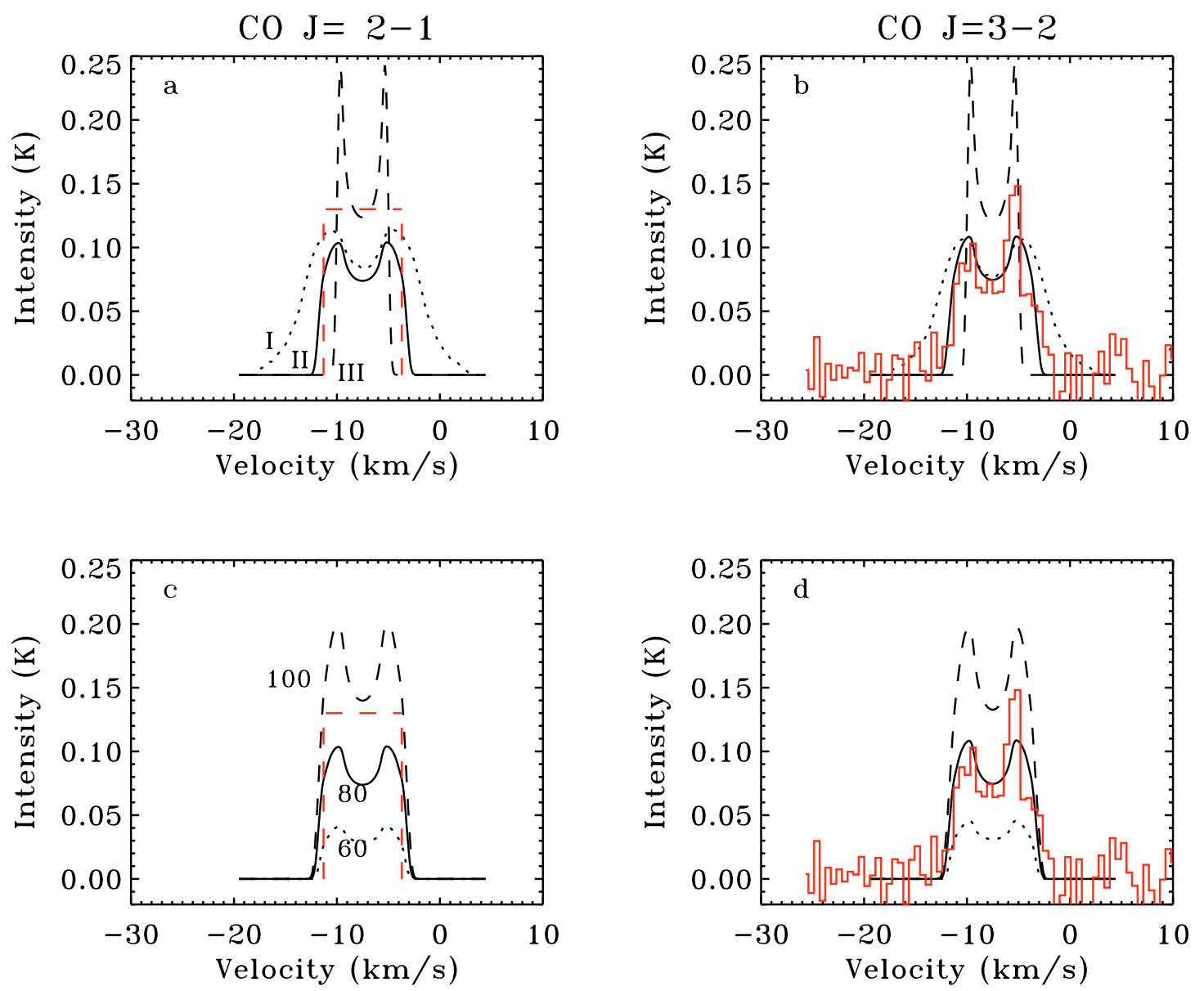

Fig. 8. CO $J=2-1$ (panels a) and c)) and $J=3-2$ (panels b) and d)) emission lines. Panels a) and b) give the best fit emission lines for the three different radial gas distributions shown in Fig. 1. The dotted line is for model I, the solid line model II, and the dashed line for model III. Panels c) and d) give the emission lines for model II with total gas masses of $60 M_{\oplus}$ (dotted line), $80 M_{\oplus}$ (solid line) and $100 M_{\oplus}$ (dashed line). The red lines give the peak intensity and full width half maximum observed by Zuckerman et al. (1995) in the left panels, and the spectrum measured by Dent et al. (2005) for the right panels.

very inefficient here. In the lower layers the abundances of $\mathrm{C}$ and $\mathrm{H}_{2}$ increase, and the gas is heated by the photoionization and photodissociation of these species, thus increasing the temperature with respect to the surface layers $(\sim 50 \mathrm{~K})$. In model III the structure is slightly more complex: in the inner parts of the disk, the cooling is inefficient due to the low densities there. In the outer disk the heating by photodissociation of $\mathrm{H}_{2}$ is very effective due to a combination of high abundances and high dissociation rate. It can be seen from Fig. 7 that removal of the PAHs from the thermal balance has little effect on the temperature structure. The temperatures found in the disk's inner edge match the $\mathrm{CO}$ rotational temperature of $190 \mathrm{~K}$ found from the $\mathrm{CO}$ vibrational bands by Brittain et al. (2003). The temperatures found here and their distribution agree well with the results of Kamp \& van Zadelhoff (2001) for the Vega disk.

Initially no temperature solution could be found in the inner disk in some models. It was found that the cooling rates for $\mathrm{C}^{+}$ and $\mathrm{O}$ were overestimated when the formulation of Tielens \& Hollenbach (1985) was used:

$\Lambda_{x}\left(v_{i j}\right)=n_{i} A_{i j} h v_{i j} \beta_{\mathrm{esc}}\left(\tau_{i j}\right) C\left(v_{i j}\right)$

where $\Lambda_{x}$ is the cooling rate by species $x, n_{i}$ is the population density of species $x$ in level $i, A$ is the spontaneous transition probability, $\beta_{\text {esc }}$ is the escape probability and $C\left(v_{i j}\right)$ is a correction factor for the infrared background radiation from hot dust and the cosmic microwave background radiation. In the Leiden PDR code UV pumping of the fine structure levels of $\mathrm{C}^{+}$ and $\mathrm{O}$ is also included in calculating the populations, and the above formula becomes invalid for very high UV fields. In the inner disk, the UV pumping becomes the dominant process for populating these levels, and as a result the cooling rates were too large. This was solved by formulating the cooling rate as

$\Lambda_{x}\left(v_{i j}\right)=h v_{i j}\left(n_{j} k_{j i}-n_{i} k_{i j}\right)$

where $n_{j}$ and $n_{i}$ are the populations of the lower and upper levels, respectively, and $k_{j i}$ and $k_{i j}$ are the collisional excitation and deexcitation rates. A stable temperature solution could be reached using this method. In the absence of UV pumping this reduces to the previous formulation.

\section{Constraining the gas mass}

\subsection{CO lines}

Figure 8 shows the predicted $\mathrm{CO} J=2 \rightarrow 1$ and $J=3 \rightarrow 2$ submillimeter lines for 5 different models: the best fitting gas masses for the radial gas distributions I, II and III (see Fig. 1), and two additional values of the gas mass for the preferred radial distribution II. The emission line profiles were calculated with the 2-dimensional radiative transfer code by 

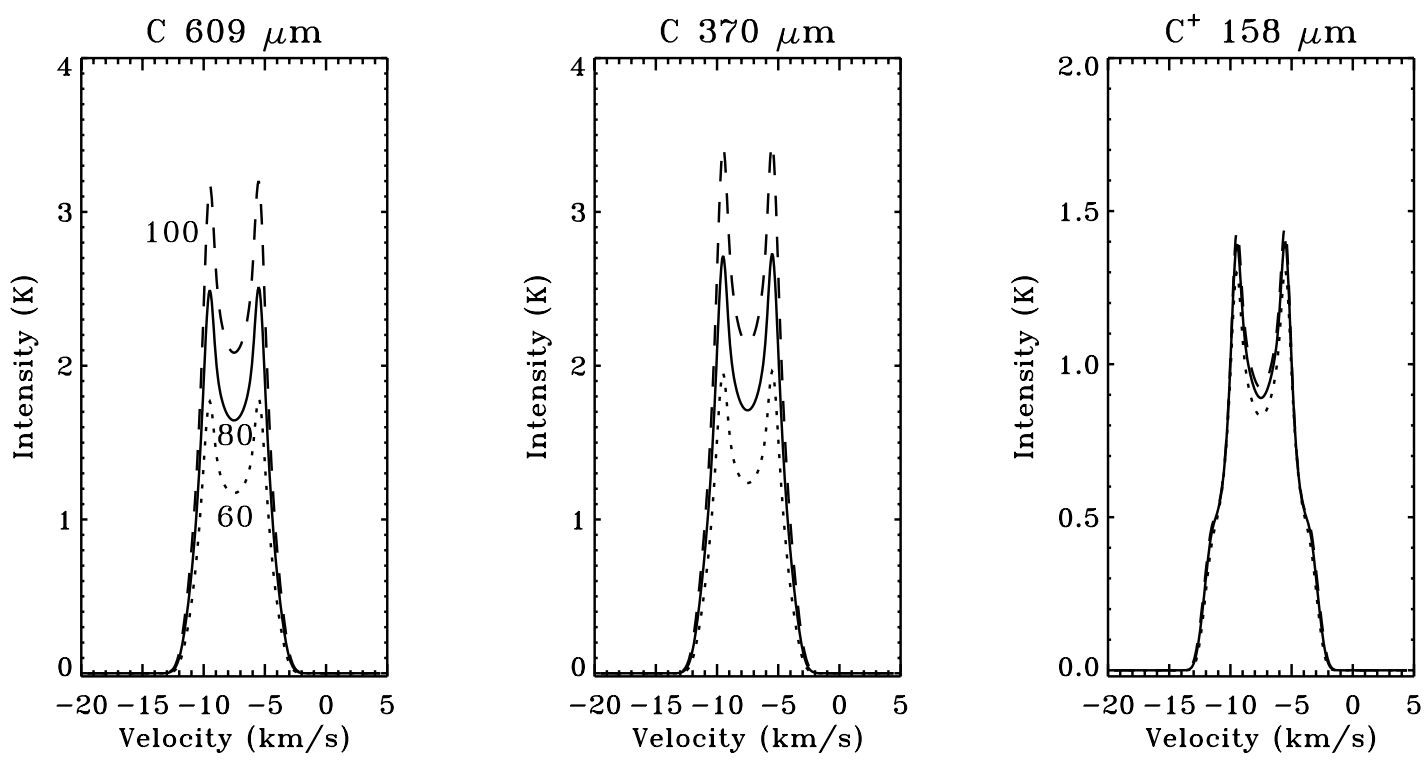

Fig. 9. The $\mathrm{C}$ and $\mathrm{C}^{+}$fine structure lines for $M_{\text {gas }}=60 M_{\oplus}$ (dotted line), $80 M_{\oplus}$ (solid line) and $100 M_{\oplus}$ (dashed line).

Hogerheijde \& van der Tak (2000). For these calculations, a distance to the HD 141569 system of 99 pc was used, and the disk is assumed to have an inclination of $55^{\circ}$ (Mouillet et al. 2001). The results are convolved with a beam of 11 arcsec for the $2 \rightarrow 1$ line (IRAM $30 \mathrm{~m}$ at $230 \mathrm{GHz}$ ), and of 14 arcsec for the $3 \rightarrow 2$ line (JCMT $346 \mathrm{GHz}$ ). Of the former line only the peak intensity and FWHM are known; of the latter, the model results are compared directly to the observed spectrum. The computational grid used in the radiative transfer code consists of 100 cells in the radial direction and 11 cells in the vertical direction.

It can be seen that the radial distribution of the gas mainly influences the widths of the lines investigated here. Distribution I gives line profiles that are too broad to fit the observations, while those of distribution III are too narrow. The intermediate distribution II fits the linewidths nicely. Using this radial distribution the emission lines were calculated for several values of the total gas mass. The strong dependence of the $\mathrm{CO}$ abundance on the gas mass shown in Fig. 4 is reflected in the emission lines, where the peak intensity changes with a factor of 4 for less than a factor of 2 in mass. It can be seen that the $80 M_{\oplus}$ model gives a good fit to the CO $J=2 \rightarrow 1$ and $J=3 \rightarrow 2$ emission lines. The model predicts peak line intensities of 0.82 and $0.78 \mathrm{~K}$ for the $J=4-3$ and $J=6-5$ lines in a $10^{\prime \prime}$ beam. The model cannot account for the sharp peak seen in the red part of the $J=3-2$ line, however. This peak is likely due to asymmetries in the disk structure, which can only be investigated by a 3 -dimensional disk model and a $3 \mathrm{D}$ radiative transfer code.

There are several uncertainties in the derived gas mass of $80 M_{\oplus}$ : first, the elemental abundance of gas-phase carbon is uncertain by a factor of a few since the fraction of carbon locked in grains is not known. Also, changes in other elemental abundances, and uncertainties in the PAH distribution and chemical rate coefficients affect the $\mathrm{CO}$ chemistry and thus the intensity of the CO lines in relation to the gas mass. Second, the CO chemistry is very sensitive to the stellar radiation field. If the star has a higher flux in the 912-1100 A range this would directly affect the abundances of $\mathrm{C}$ and $\mathrm{CO}$. Third, the assumption that $\mathrm{H}_{2}$ can form on PAHs is critical to the chemistry presented here. If the formation of $\mathrm{H}_{2}$ is less efficient than assumed here it would reduce the abundance of all molecules in the disk, both because $\mathrm{H}_{2}$ is is a progenitor molecule for many species and because of the shielding to UV photons it supplies. For comparison of results obtained with a completely independent chemical code (Kamp \& van Zadelhoff 2001), an uncertainty in the gas mass of a factor of a few is estimated.

The effect of disk inclination on the line profiles is limited. Spectra of the CO 3-2 line for distribution II with a gas mass of $80 M_{\oplus}$ with inclinations of $50^{\circ}$ and $60^{\circ}$ are very similar to the spectrum shown in Fig. 8 and show equally good agreement with the observed spectrum. Since a $5^{\circ}$ change in inclination would indicate a morphological eccentricity of the disk of $\sim 0.45$, the value of $55^{\circ}$ by Mouillet et al. (2001) is retained.

The models presented here confirm the presence of gas in the inner disk previously noted by Zuckerman et al. (1995), Brittain et al. (2003) and Dent et al. (2005). Furthermore, the presence of vibrationally excited CO at temperatures of 150-200 K found by Brittain et al. (2003) is matched by model II; model I has too high temperatures in the inner disk, while model III only has CO in the outer disk where the radiation field is too low to excite the vibrational levels of $\mathrm{CO}$ and the temperatures are too low to match the observations.

\subsection{Other emission lines}

The predicted [C I] and [C II] fine structure lines at 609, 370 and $158 \mu \mathrm{m}$ are shown in Fig. 9. The intensities were calculated assuming radial distribution II, and assuming the beam is exactly the size of the disk ( 10 arcsec). Although the intensities of the [C I] lines change less dramatically with disk mass than the CO lines they are still a good - and probably more direct (see Fig. 4) - indicator of the total disk mass. The [C II] line on the other hand depends only very weakly on the disk mass; while there is more carbon in disks of higher mass, the fraction of ionized carbon is smaller.

It should be noted that the $\mathrm{C}$ abundance and $[\mathrm{C} \mathrm{I}]$ intensity are sensitive to the UV intensity at $\lambda<1100 \AA$. In particular, the $\mathrm{C}^{+} / \mathrm{C}$ ratio is increased and the $[\mathrm{CI}]$ intensity decreased if the UV intensity drops less steeply at the shortest wavelengths. In that case, the CO self-shielding can be maintained with an only slightly higher gas mass while the relative importance of 
$\mathrm{C}$ photoionization to $\mathrm{CH}$ photodissociation is decreased, leading to a lower $\mathrm{C}$ abundance.

\subsection{Gas/dust ratio}

The spatial dust distribution considered in this paper has a total mass of $\sim 2.2 M_{\oplus}$ for grains with sizes $1 \mu \mathrm{m}<a<1 \mathrm{~cm}$. Together with the gas mass of $80 M_{\oplus}$ this would give an overall gas/dust mass ratio of 36 , which cannot be distinguished from the interstellar value of 100 given the uncertainties in the gas mass. This may indicate that gas and dust are dissipated on similar timescales. However, the upper limit of the grain size distribution is not well constrained, and the total dust mass depends strongly on this parameter. Furthermore, it is uncertain what part (if any) of the dust population is debris (i.e. the product of collisions between meter-sized bodies) and what part is "first generation" aggregates. Thus the derived gas/dust ratio has only limited meaning.

\subsection{Second generation gas?}

There is a possibility that the gas in an evolved disk such as that around HD 141569A, is entirely hydrogen-poor, second generation gas, i.e. gas originating from evaporating solid bodies instead of the original interstellar cloud. The CO photodissociation timescale is short, $\sim 10^{3}$ years (see Sect. 3.1). In order to reproduce the observed $\mathrm{CO}$ mass of $4 \times 10^{-4} M_{\oplus}, \mathrm{CO}$ has to evaporate from comets (or similar solid bodies) at a rate of $4 \times$ $10^{-7} M_{\oplus} \mathrm{yr}^{-1}$. If one takes a mass of $10^{18} \mathrm{~g}\left(2 \times 10^{-10} M_{\oplus}\right)$ and a CO mass fraction of $10 \%$ as typical numbers for solar system comets (see Delsemme 1985) this translates into a required evaporation rate of $2 \times 10^{4}$ comets per year, which seems too high to be realistic.

\section{Conclusions}

A model of the HD 141569A disk has been constructed which calculates the chemistry and gas temperature in a quasi 2-dimensional formalism and predicts the resulting emission lines. The main conclusions are as follows:

- A gas distribution with a total mass of $80 M_{\oplus}$ starting at $80 \mathrm{AU}$ from the central star produces the best fit to the observed CO emission lines for this object. Further observations of gas-phase lines will be needed to corroborate this result. The gas distribution can be tested through spatially resolved $\mathrm{CO}$ observations. The [C I] fine-structure lines are good tracers of gas mass, but the [C II] fine-structure line shows only a very weak variation with disk mass.

- The CO chemistry of the outer disk is very sensitive to the total gas mass due to the coupled effects of high densities (which drives carbon to its molecular form) and shielding of the stellar and interstellar radiation fields.

- The interstellar radiation field plays a significant role in the dissociation of $\mathrm{H}_{2}$ and $\mathrm{CO}$, because the lines through which these molecules dissociate become optically thick toward the central star.

- PAHs are found to be very important in the chemistry. Because the grains in this disk are large, the most efficient way to form $\mathrm{H}_{2}$ is via PAHs, and therefore they affect the formation of all molecules.

- Carbon is found to be predominantly in neutral atomic form due a combination of efficient self-shielding of carbon photoionization and rapid photodissociation of chemical precursors of $\mathrm{CO}$ by the stellar radiation field. This conclusion depends strongly on the shape of the stellar radiation field at $\lambda<1100 \AA$.

- The current model reproduces the CO $J=3 \rightarrow 2$ line profile observed by Dent et al. (2005) quite well. The sharp peak at the red side of the spectrum is not reproduced due to the cylindrical symmetry inherent in the model. Also the model uses a azimuthally averaged dust distribution while the real distribution is noticeably sharper, which also explains the shallower profile. The current model ignores the possibility of a clumpy gas distribution, which would also result in sharper profiles.

The calculations of the gas mass of the HD 141569 disk presented here indicate that transitional disks retain a fraction of their original gas content after a few Myr. The remaining gas is not enough to form giant planets, but mechanisms exist for planets to form on shorter timescales than the age of the disk (e.g. Pollack et al. 1996). It is expected that the remaining gas has a strong influence on the dust dynamics in the disk.

Acknowledgements. The authors are grateful to Xander Tielens for helpful discussions of the relevant heating rates, to David Mouillet for the deprojected HST image in Fig. 2, and to Bill Dent for communicating his observational data in Fig. 8. They thank Michiel Hogerheijde and Floris van der Tak for the use of their $2 \mathrm{D}$ radiative transfer code. This work was supported by a Spinoza grant from the Netherlands Organisation for Scientific Research and by the European Community's Human Potential Programme under contract HPRN-CT-2002-00308, PLANETS.

\section{References}

Augereau, J.-C., Lagrange, A. M., Mouillet, D., \& Ménard, F. 1999, A\&A, 350, L51

Augereau, J.-C., \& Papaloizou, J. C. B. 2004, A\&A, 414, 1153

Black, J. H., \& van Dishoeck, E. F. 1987, ApJ, 322, 412

Boccaletti, A., Augereau, J.-C., Marchis, F., \& Hahn, J. 2003, ApJ, 585, 494

Brittain, S. D., Rettig, T. W., Simon, T., et al. 2003, ApJ, 588, 535

Clampin, M., Krist, J. E., Ardila, D. R., et al. 2003, AJ, 126, 385

Delsemme, A. H. 1985, PASP, 97, 861

Dent, W. R. F., Greaves, J. S., \& Coulson, I. M. 2005, MNRAS, 359, 663

Draine, B. T. 1978, ApJS, 36, 595

Draine, B. T., \& Bertoldi, F. 1996, ApJ, 468, 269

Hauschildt, P. H., Allard, F., \& Baron, E. 1999, ApJ, 512, 377

Hogerheijde, M. R., \& van der Tak, F. F. S. 2000, A\&A, 362, 697

Jonkheid, B., Faas, F. G. A., van Zadelhoff, G.-J., \& van Dishoeck, E. F. 2004, A\&A, 428, 511

Kamp, I., \& van Zadelhoff, G.-J. 2001, A\&A, 373, 641

Kamp, I., van Zadelhoff, G.-J., van Dishoeck, E. F., \& Stark, R. 2003, A\&A, 397,1129

Klahr, H., \& Lin, D. N. C. 2005, ApJ, 632, 1113

Li, A., \& Lunine, J. I. 2003, ApJ, 594, 987

Mathis, J. S., Rumpl, W., \& Nordsieck, K. H. 1977, ApJ, 217, 425

Merín, B., Montesinos, B., Eiroa, C., et al. 2004, A\&A, 419, 301

Mouillet, D., Lagrange, A. M., Augereau, J.-C., \& Ménard, F. 2001, A\&A, 372, L61

Pollack, J. B., Hubickyj, O., Bodenheimer, P., et al. 1996, Icarus, 124, 62

Rossiter, R. A. 1943, Publ. Michigan Obs., 8, 133

Shu, F. H., Adams, F. C., \& Lizano, S. 1987, ARA\&A, 25, 23

Simon, M., \& Prato, L. 1995, ApJ, 450, 824

Spaans, M., Tielens, A. G. G. M., van Dishoeck, E. F., \& Bakes, E. L. O. 1994, ApJ, 437, 270

Sylvester, R. J., Skinner, C. J., Barlow, M. J., \& Mannings, V. 1996, MNRAS, 279,915

Tielens, A. G. G. M., \& Hollenbach, D. J. 1985, ApJ, 291, 722

van Dishoeck, E. F. 1988, in Rate Coefficients in Astrochemistry, ASSL, 146, 49 van Dishoeck, E. F., \& Black, J. H. 1988, ApJ, 334, 771

Watson, W. D. 1972, ApJ, 176, 103

Weinberger, A. J., Becklin, E. E., Schneider, G., et al. 1999, ApJ, 525, L53

Weinberger, A. J., Becklin, E. E., \& Zuckerman, B. 2004, Am. Astron. Soc. Meet. Abstracts, 205

Weinberger, A. J., Rich, R. M., Becklin, E. E., Zuckerman, B., \& Matthews, K. 2000, ApJ, 544, 937

Werner, M. W. 1970, Astrophys. Lett., 6, 81

Wyatt, M. C. 2005, A\&A, 440, 937

Zuckerman, B., Forveille, T., \& Kastner, J. H. 1995, Nature, 373, 494 